\title{
Removal of intrauterine contraception with non-visible threads: a case series
}

\author{
Elizabeth Ann Stephens
}

\section{Correspondence to}

Dr Elizabeth Ann Stephens,

Abacus Community Sexual Health Service, Merseycare NHS

Foundation Trust, Liverpool

L14AP, UK; liz.stephens@ merseycare.nhs.uk

Received 19 April 2018 Revised 11 September 2018 Accepted 18 September 2018 Published Online First

15 October 2018
Check for updates

(c) Author(s) (or their employer(s)) 2019. No commercial re-use. See rights and permissions. Published by BMJ.

To cite: Stephens EA. BMJ Sex Reprod Health

2019;45:44-46.

\section{ABSTRACT}

Objectives (1) To review management of a series of women referred for removal of intrauterine contraception (IUC) with non-visible threads.

(2) To establish whether the device was likely

to have been placed at the fundus at insertion.

(3) To document removal success rates in a

community sexual health (CSH) setting.

Study design A retrospective review of a series of 76 women seen by the author between April 2016 and October 2017 in a specialist CSH clinic for removal of IUC with non-visible threads.

Results After ultrasound scan (USS) assessment 67 women underwent a removal procedure.

Sixty-two devices (92.5\%) were successfully removed. Uterine instrumentation beyond the internal os was required in 43 removals, enabling comparison of uterine cavity length with the length of IUC and threads. Such comparison suggested 39/43 (91\%) devices were not fundal at insertion and that non-visible threads were likely to have been caused by the device moving to the fundus post-insertion under the influence of uterine contractions, leading to retraction of the threads.

Conclusions Removal of IUC with non-visible threads can be successfully done in a CSH setting with ultrasound availability. Non-fundal placement of IUC at insertion is likely to be a significant cause of non-visible threads.

\section{INTRODUCTION}

Rates of non-visible threads at the time of removal of intrauterine contraception (IUC) have been quoted as being from under $5 \%$ to $18 \% .^{12}$ Women wishing removal of a device where the threads are found to be missing should have an ultrasound scan (USS) to confirm that the device is in situ before the uterus is instrumented. ${ }^{3}$ Marchi et al reviewed the records of 14935 patients using IUC and found 750 women (5.0\%) who presented to follow-up with missing threads. ${ }^{1}$ USS showed the IUC to be in situ in $98 \%$ of

\section{Key messages}

- The findings of this study suggest that the major cause of non-visibility of intrauterine contraception (IUC) threads is non-fundal placement of devices at the time of insertion.

- Greater attention needs to be paid to IUC insertion technique to ensure fundal placement of devices at the time of insertion.

- Consideration should be given to routine ultrasound scan post insertion to confirm fundal placement.

those cases, while $1.2 \%$ had been expelled and $0.7 \%$ had perforated.

There are very few studies that have looked at the reasons why threads are not visible. Swenson $\mathrm{et} \mathrm{al}^{4}$ postulated one cause as a device being inadvertently pulled down into the lower uterine cavity on withdrawal of the inserter, and subsequently moving towards the fundus under the influence of uterine contractions, leading to retraction of the threads from the cervical os. They tested this hypothesis by measuring the total length of the device and threads and suggested that a combined measurement of $<7.5 \mathrm{~cm}$ meant that the device could not have been fundal at insertion. This study aimed to explore this hypothesis further and to identify the success rate of removal of IUC with non-visible threads in a community setting.

\section{METHODS}

This was a retrospective review of a series of 76 women referred for removal of IUC with non-visible threads and seen by the author in the Abacus specialist city-centre Community Sexual Health (CSH) clinic between 1 April 2016 and 31 October 2017. Sources of referral were from within 
the Abacus service and externally from general practitioners and other CSH clinics. Data were collected on parity, type of device and type of instrument used for removal. The uterine cavity length was measured only if instrumentation beyond the internal os was required, that is, when Spencer Wells forceps, or an Emmett thread retriever inserted to the level of the internal os, had failed to retrieve the threads or remove the device. If the Emmett thread retriever failed to retrieve threads from the cervical canal it was then inserted to the uterine fundus and following its removal, with or without the device or threads, it was placed next to a graduated uterine sound to record the overall uterine cavity length. If the device was not removed using the thread retriever, Hartmann crocodile forceps, inserted into the uterine cavity, were then used. Following IUC removal, the length from the top of the device to the end of the threads was recorded if the uterine length had needed to be measured. The device lengths were divided into two groups: total length greater than the uterine length plus $1 \mathrm{~cm}$ and total length less than the uterine length plus $1 \mathrm{~cm}$.

\section{Patient and public involvement}

As this was a retrospective review of cases, patients were not involved in the design of this study.

\section{RESULTS}

Seventy-six women were seen for IUC removal with non-visible threads from April 2016 to October 2017.
During that time period a total of 1466 IUC devices were removed at Abacus overall: 799 intrauterine systems (IUSs) and 667 intrauterine devices (IUDs).

Of the 76 women, the IUD/IUS was not seen in four women on initial USS. In one case the device was seen in the uterine cavity at a subsequent USS. In the other three women the IUD/IUS was not seen on subsequent abdominal X-ray, confirming expulsion; there were no perforations. The remaining 72 devices were intrauterine (figure 1).

\section{Characteristics of women with IUC in the uterine cavity}

Three-quarters of the women $(54 / 72 ; 75 \%)$ were parous. Of women having IUC inserted at Abacus during the same period, $57 \%$ were parous and $43 \%$ nulliparous.

Forty-six (64\%) of the 72 devices were IUSs and 26 $(36 \%)$ were IUDs. Of IUC inserted in the same time period, 45\% were IUSs and 55\% IUDs.

Of the IUDs successfully removed, one was a Chinese ring (a threadless device), three were Multiload, 13 were NeoSafeT380 and four were TT380Slimline.

\section{Outcome of attempted removal}

Threads were visible at the external os in two cases and IUC removal was therefore routine. Two women chose to continue with their devices and one was advised to defer the removal procedure as she had had sexual intercourse in the preceding 7 days.

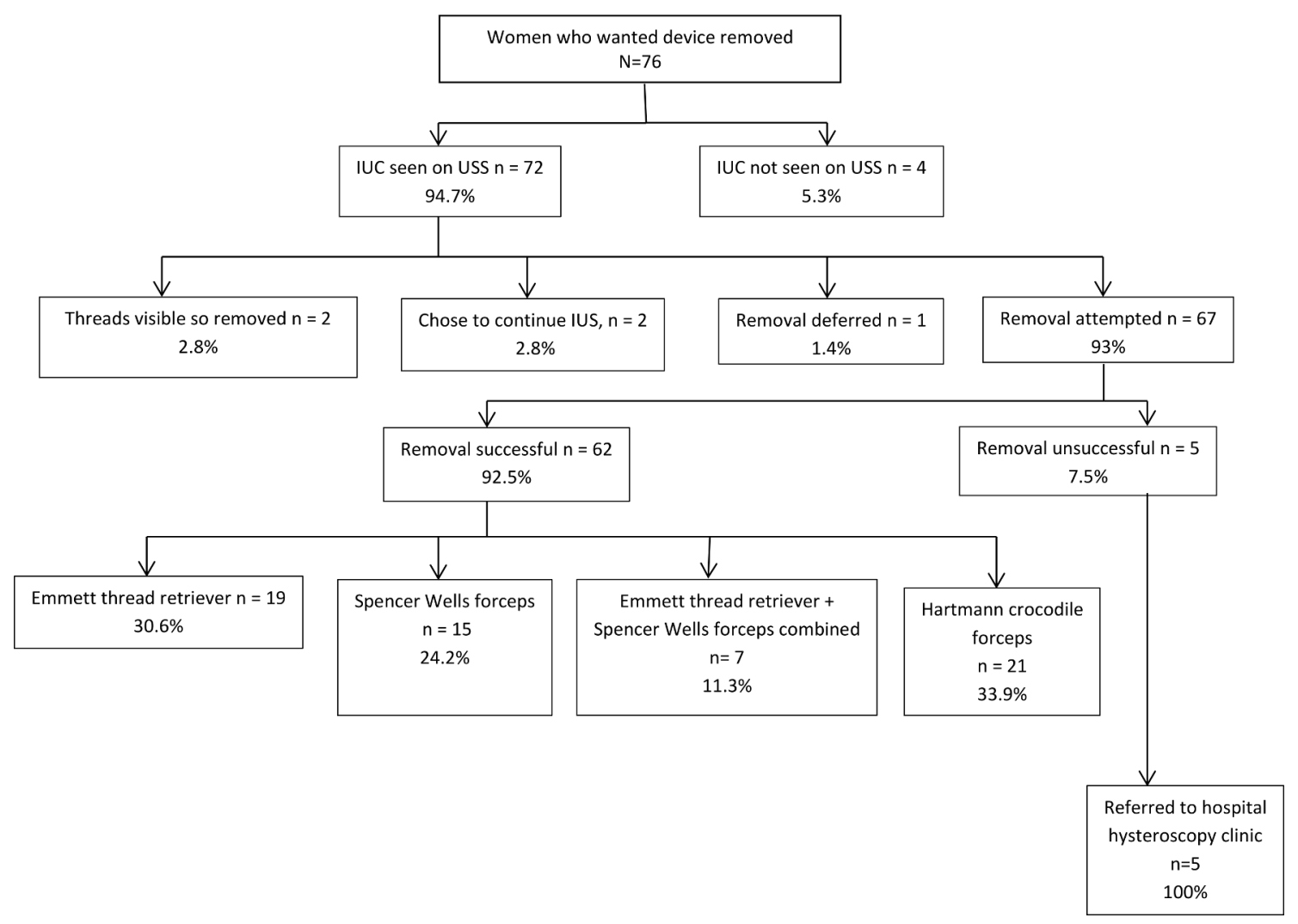

Figure 1 Outcome of 76 women seen for intrauterine contraception (IUC) removal with non-visible threads. USS, ultrasound scan. 
Most of the remainder of the devices $(62 / 67 ; 92.5 \%)$ were removed successfully in the clinic:

- 15 (24\%) with Spencer Wells forceps to grasp threads in the cervical canal

- $4(7 \%)$ with Emmett thread retriever to retrieve threads from the cervical canal

- 15 (24\%) with Emmett thread retriever passed beyond the internal os

- 7 (11\%) with a combination of Emmett thread retriever beyond the internal os and Spencer Wells forceps

- 21 (34\%) with Hartmann crocodile forceps inserted to the uterine cavity.

Five attempted IUD removals were unsuccessful. All were referred to secondary care and the devices were removed at hysteroscopy. Four of the devices were embedded in the myometrium; three of those women were parous.

\section{Measurements}

Uterine instrumentation beyond the internal os was required in 43/62 removals (67\%). In 39/43 (91\%), the measurement from the top of the device to the end of threads was less then than the uterine sounding length plus $1 \mathrm{~cm}$. In the remainder, $4 / 43(9 \%)$, that measurement was $>1 \mathrm{~cm}$ longer than the uterine sounding length

\section{DISCUSSION}

In $91 \%$ of women where measurements were recorded (39/43), the measurement from the top of device to the end of threads was less than the uterine sounding length plus $1 \mathrm{~cm}$, suggesting that the device may not have been fundal at insertion. The cause of the non-visible threads was likely to have been movement of the device towards the fundus after insertion due to uterine contractions or the threads having been cut very short at insertion, or both.

It is not possible to identify the rates of non-visible threads at the time of removal from this review as it does not include cases seen by other clinicians within our service.

This study expands on the findings of Swenson et $a l^{4}$ in that it is a larger sample and includes IUDs as well as IUSs and nulliparous as well as parous women. Non-fundal placement of IUC at the time of insertion and subsequent movement of the device towards the fundus appears to be a major cause of non-visible threads. Potential causes of non-fundal placement include inaccurate sounding of the uterine cavity length and correct sounding but inadvertent pulling of the device into the lower uterine cavity on withdrawal of the inserter. Nulliparity does not appear to have a bearing on the likelihood of non-visible threads.

Performing an ultrasound scan immediately after IUC insertion could detect devices which are not fundal, allowing removal and repeat insertion, thereby reducing the likelihood of subsequent non-visible threads,

A number of studies have advocated ultrasound-guided removal of IUC with non-visible threads. ${ }^{2} 5$ Our findings suggest that skilled retrieval with an Emmett thread retriever, Spencer Wells forceps or Hartmann crocodile forceps after USS to confirm that the device is in place is nearly always successful, and that ultrasound monitoring of the retrieval procedure is therefore only rarely necessary.

Acknowledgements Terry Williams, Abacus Sexual Health Directorate, carried out data analysis.

Contributors The author saw all the patients in clinic, conducted the review of their cases, carried out data collection, interpretation of results and wrote up the report. Mr Terry Williams carried out the data analysis and is listed in the acknowledgement section.

Funding The authors have not declared a specific grant for this research from any funding agency in the public, commercial or not-for-profit sectors.

Competing interests None declared.

Patient consent Not required.

Provenance and peer review Not commissioned; externally peer reviewed.

\section{REFERENCES}

1 Marchi NM, Castro S, Hidalgo MM, et al. Management of missing strings in users of intrauterine contraceptives. Contraception 2012;86:354-8.

2 Moro F, Knez J, Pateman K, et al. Ultrasound-guided retrieval of lost intrauterine devices using very fine grasping forceps: a case series. J Fam Plann Reprod Health Care 2015;41:205-9.

3 Faculty of Sexual \& Reproductive Healthcare Clinical Effectiveness Unit, 2015. Intrauterine contraception. https:// www.fsrh.org/standards-and-guidance/documents/ceuguidancei ntrauterinecontraception/ (accessed 25 Jan 2018).

4 Swenson C, Royer PA, Turok DK, et al. Removal of the LNG IUD when strings are not visible: a case series. Contraception 2014;90:288-90.

5 Verma U, Astudillo-Dávalos FE, Gerkowicz SA. Safe and costeffective ultrasound guided removal of retained intrauterine device: our experience. Contraception 2015;92:77-80. 